\title{
Stellar yields and abundances: new directions from planetary nebulae
}

\author{
Maria Lugaro ${ }^{1,2}$, Amanda I. Karakas ${ }^{2,3}$, Marco Pignatari ${ }^{4,5}$ and \\ Carolyn L. Doherty ${ }^{1}$ \\ ${ }^{1}$ Konkoly Observatory, Research Centre for Astronomy and Earth Sciences, \\ Hungarian Academy of Sciences, H-1121 Budapest, Hungary \\ email: maria.lugaro@csfk.mta.hu, carolyn.doherty@csfk.mta.hu \\ ${ }^{2}$ Monash Centre for Astrophysics, School of Physics and Astronomy, Monash University, VIC \\ 3800, Australia \\ email: amanda.karakas@monash.edu \\ ${ }^{3}$ Research School of Astronomy and Astrophysics, Australian National University, Canberra, \\ ACT 2611, Australia \\ ${ }^{4}$ E. A. Milne Centre for Astrophysics, University of Hull, Cottingham Road Hull HU6 7RX \\ United Kingdom \\ email: m.pignatari@hull.ac.uk \\ ${ }^{5}$ The NuGrid collaboration (www.nugridstars.org)
}

\begin{abstract}
Planetary nebulae retain the signature of the nucleosynthesis and mixing events that occurred during the previous AGB phase. Observational signatures complement observations of AGB and post-AGB stars and their binary companions. The abundances of the elements heavier than iron such as $\mathrm{Kr}$ and Xe in planetary nebulae can be used to complement abundances of $\mathrm{Sr} / \mathrm{Y} / \mathrm{Zr}$ and $\mathrm{Ba} / \mathrm{La} / \mathrm{Ce}$ in $\mathrm{AGB}$ stars, respectively, to determine the operation of the slow neutron-capture process (the $s$ process) in AGB stars. Additionally, observations of the Rb abundance in Type I planetary nebulae may allow us to infer the initial mass of the central star. Several noble gas components present in meteoritic stardust silicon carbide ( $\mathrm{SiC})$ grains are associated with implantation into the dust grains in the high-energy environment connected to the fast winds from the central stars during the planetary nebulae phase.
\end{abstract}

Keywords. nuclear reactions, nucleosynthesis, abundances, stars: AGB and post-AGB, stars: abundances, stars: chemically peculiar

\section{Introduction}

The evolutionary track of the life of a star of $2 M_{\odot}$ after core $\mathrm{H}$ exhaustion proceeds to the red giant phase, until core He burning stars. After core He exhaustion, the star becomes a red giant again and climbs the asymptotic giant branch (AGB). During the AGB phase, the stars loses most of its envelope via strong stellar winds, until mainly the $\mathrm{C}-\mathrm{O}$ degerate core is left, with a thin layer of $\mathrm{H}\left(\sim 10^{-3} M_{\odot}\right)$ still burning. At this point the star travels back in the H-R diagram towards hotter temperatures. If it becomes hot enough before all the material expelled in the previous AGB phase is dispersed, the UV photons from the central star illuminate the circumstellar material producing a planetary nebula. It follows that in planetary nebulae we observe the chemical abundances produced during the AGB phase.

During the AGB the structure of the star can be described by two components: the entended convective H-rich envelope and the compact core. The core is hot and dense, and nuclear reactions can occur there that produce and modify the chemical abundances. Two burning shells are present: the $\mathrm{H}$ and the He burning shells. These shells act alternately, 
with recurrent episodes of He burning driving convective motions (a thermal pulse, TP) in the whole region in-between the two shells (the intershell). As the star expands and cools down, $\mathrm{H}$ burning is extinguished, the TP ceases, and He continues to burn radiatively at the bottom of the intershell. At this point the base of the convective envelope may sink deep inside the intershell (third dredge-up, TDU) and carry to the stellar surface the products of $\mathrm{H}$ and He burning. After the TDU, He burning extinguishes and $\mathrm{H}$ burning starts up again. This cycle of $\mathrm{H}$ burning, TP, and TDU, repeats until the envelope mass is lost. The material brought to stellar surface is carried into the surroundings by the AGB winds and contributes to making up the chemical enrichment of the Galaxy. For reviews on AGB stars, see Herwig (2005) and Karakas \& Lattanzio (2014).

Four main chemical signatures result from the AGB evolution:

(a) During the TPs, partial He burning makes carbon, and not much oxygen, in the intershell and stars in the mass range roughly 1.5 to $4 M_{\odot}$ (depending on the initial composition) can eventually become the observed C-rich stars (see, e.g., the middle panels of Figs. 2-4 of Karakas \& Lugaro 2016).

(b) The base of the convective envelope can be hot enough to drive proton captures on the isotopes of the elements from $\mathrm{C}$ to $\mathrm{Al}$ (hot bottom burning, HBB). They transform, for example, $\mathrm{C}$ into $\mathrm{N}$ in stars of initial mass larger than roughly $4 M_{\odot}$ (see, e.g., the bottom panels of Figs. 2-4 of Karakas \& Lugaro 2016).

(c) The ingestion of H-burning ashes in the TPs results in the production of specific rare isotopes, such as ${ }^{19} \mathrm{~F}$ and ${ }^{22} \mathrm{Ne}$ (e.g., Lugaro et al. 2004; Abia et al. 2015).

(d) Finally, AGB stars are the site of origin of half of the cosmic abundances of the elements heavier than iron, via the operation of the slow neutron capture process (the $s$ process) in the intershell.

The last of these four points will be discussed in more detail in the present paper, together with implications from the observations of planetary nebulae.

\subsection{The $s$ process}

Because of their high number of protons $(>26)$ the elements heavier than Fe have a large Coulomb barrier and can be produced only by capturing neutrons. The $s$ process occurs typically in hydrostatic conditions at relatively low neutron densities (typical $10^{8} \mathrm{n} / \mathrm{cm}^{3}$, but up to $10^{13} \mathrm{n} / \mathrm{cm}^{3}$ in the more massive AGB stars). The path of neutron captures proceeds mostly through stable nuclei, since unstable nuclei decay to their corresponding isobar before they can capture another neutron.

Along the path of stable nuclei, the flux of neutron captures encounters three bottlenecks corresponding to the magic number of neutrons $\mathrm{N}=50,82,126$ (Fig. 1). Nuclei with such numbers of neutrons are relatively more stable against neutron captures than other nuclei, and tend to accumulate until the neutron flux reaches a large enough value to make the probability of neutron captures on these nuclei significant. The total, timeintegrated neutron flux is the crucial quantity that determines if the bottlenecks are bypassed and if the production flux can proceed to the next bottleneck. The bottleneck nuclei determine the final $s$-process distribution and correspond to the so-called first $s$ process peak - or light s-process elements $(l s)$ with $\mathrm{N}=50, \mathrm{Sr}, \mathrm{Y}, \mathrm{Zr}$; second $s$-process peak - or heavy s-process elements $(h s)$ with $\mathrm{N}=82$, Ba, La, Ce; and third $s$-process peak at $\mathrm{N}=126(\mathrm{~Pb})$, which can only accumulate since the $s$-process path ends there. The traditional $s$-process indexes are defined as $[\mathrm{ls} / \mathrm{Fe}]=([\mathrm{Sr} / \mathrm{Fe}]+[\mathrm{Y} / \mathrm{Fe}]+[\mathrm{Zr} / \mathrm{Fe}]) / 3$ and $[\mathrm{hs} / \mathrm{Fe}]=([\mathrm{Ba} / \mathrm{Fe}]+[\mathrm{La} / \mathrm{Fe}]+[\mathrm{Ce} / \mathrm{Fe}]) / 3$; although other choices have also been used in the literature (see discussion in Lugaro et al. 2012). 


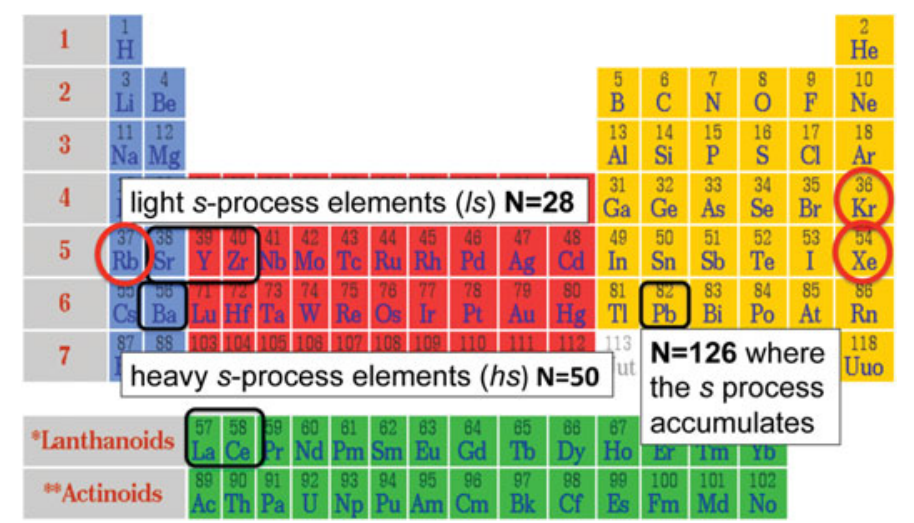

Figure 1. Periodic table of the elements with the locations of the first, second and third $s$ process peaks marked along with the corresponding neutron numbers. Also highlighted are the noble gases $\mathrm{Kr}$ and $\mathrm{Xe}$ and the element $\mathrm{Rb}$, which can be observed in planetary nebulae.

\subsection{The s-process in $A G B$ stars}

In the intershell of AGB stars free neutron are produced by two reactions. The ${ }^{13} \mathrm{C}(\alpha, \mathrm{n}){ }^{16} \mathrm{O}$ reaction is the main neutron surce in AGB stars of mass lower than roughly $4 M_{\odot}$. A region rich in ${ }^{13} \mathrm{C}$, the ${ }^{13} \mathrm{C}$ pocket, is assumed to form via mixing of protons from the envelope into the intershell at the deepest extent of the TDU (Gallino et al. 1998; Goriely \& Mowlavi 2000; Lugaro et al. 2003b; Cristallo et al. 2009). The protons react with the abundant ${ }^{12} \mathrm{C}$ to produce ${ }^{13} \mathrm{C}$ via ${ }^{12} \mathrm{C}(\mathrm{p}, \gamma){ }^{13} \mathrm{~N}\left(\beta^{+}\right){ }^{13} \mathrm{C}$. The ${ }^{13} \mathrm{C}$ pocket controls the total number of neutrons and thus the observed $s$-process distribution, i.e., the [hs/ls] ratio. The absolute $s$-process abundances $[\mathrm{ls} / \mathrm{Fe}]$ and $[\mathrm{hs} / \mathrm{Fe}]$ are controlled also by the TDU and other dilution effects. For example, binary transfer plays a crucial role in interpreting the data derived from an AGB star companion (a so-called extrinsic s-process enhanced star), rather than an AGB star itself (an intrinsic s-process enhanced star). Because both the formation of the ${ }^{13} \mathrm{C}$ pockets and the effects of stellar rotation and magnetic fields on the neutron flux are very uncertain, observations of [hs $/ \mathrm{ls}]$ represent one of a few viable ways to understand the $s$ process in low-mass AGB stars.

For AGB stars more massive than roughly $4 M_{\odot}$, the main source of neutrons is instead the ${ }^{22} \mathrm{Ne}(\alpha, \mathrm{n})^{25} \mathrm{Mg}$ reaction, which operates at higher temperatures (above 300 MK) inside the convective TPs. This neutron source produces a lower total number of neutrons but a higher neutron density than the ${ }^{13} \mathrm{C}$ source. As such, it controls the possible operation of branching points, introducing deviations from the main $s$-process path. For instance, the abundance ratio $[\mathrm{Rb} / \mathrm{Zr}]$ is an indicator of the neutron density and of the activation of the ${ }^{22} \mathrm{Ne}$ source. The production of $\mathrm{Rb}$ is much increased when the neutron magic isotope ${ }^{87} \mathrm{Rb}(\mathrm{N}=50)$ is reached by the $s$-process path via the activation of the branching points at the unstable ${ }^{85} \mathrm{Kr}$ and ${ }^{86} \mathrm{Rb}$ (Abia et al. 2001; van Raai et al. 2012). Large uncertainties are present in the operation of the ${ }^{22} \mathrm{Ne}$ neutron source including the rate of the neutron source reaction, the efficiency of the TDU, and the mass loss rate in massive AGB stars. The difficulty in constraining the operation of this neutron source using observations is that the $[\mathrm{Rb} / \mathrm{Zr}]$ ratio in massive AGB stars is highly dependent on the model atmosphere used to analyse the spectra (Zamora et al. 2014). Independent constraints are crucial to settle the question of how much $\mathrm{Rb}$ is produced by massive AGB stars. 


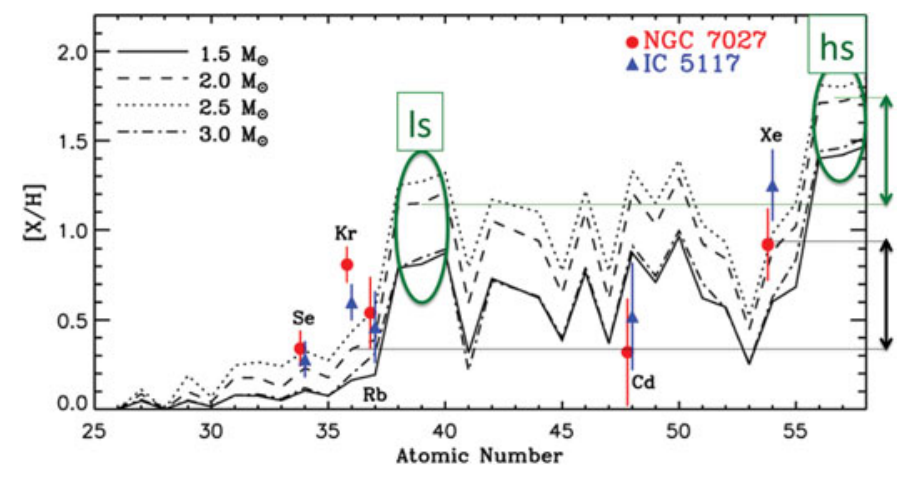

Figure 2. Figure adapted from Sterling et al. (2016) (CAAS. Reproduced with permission) showing the comparison between abundances observed in two planetary nebulae (red and blue circle symbols, with error bars) and results from models of the $s$ process in AGB stars from the FRUITY database (Cristallo et al. 2015) for different masses and metallicity $[\mathrm{Fe} / \mathrm{H}]=-0.37$. Also indicated are the location of the first and second $s$-process peaks, labelled as ls and hs, respectively. The green and black arrows on the right side of the figure measure the $[\mathrm{hs} / \mathrm{ls}]$ and the $[\mathrm{Xe} / \mathrm{Kr}]$ ratios, respectively, predicted by the $2 M_{\odot}$ model. It can be seen that $[\mathrm{hs} / \mathrm{ls}] \simeq[\mathrm{Xe} / \mathrm{Kr}]$.

\section{New directions}

Observations of the elements heavier than Fe in planetary nebulae (see Sterling et al., these proceedings) provide us complementary indicators and independent constraints for the $s$-process in AGB stars. As it can be seen from Fig. 1 the noble gases $\mathrm{Kr}$ and Xe, which are observable in planetary nebulae, are located very close to the first and second $s$ process peaks, respectively. Interestingly, only a small fraction of the cosmic abundances of these noble gases are produced by the $s$-process in AGB stars (Arlandini et al. 1999; Bisterzo et al. 2014): Xe is a typical element made by the rapid neutron captures in explosive environments (Thielemann et al. 2011). Krypton, on the other hand, is mostly produced in massive stars by a multitude of processes, including the weak $s$ process and different explosive mechanisms (e.g., Roberts et al. 2010; Pignatari et al. 2016, and references therein).

Nevertheless, the $s$ process in AGB stars contributes roughly $30 \%$ and $15 \%$ of the solar Kr and Xe, respectively (Bisterzo et al. 2014). This production, although much lower in absolute terms, follows the production of the $s$-process first and second peaks. This means that effectively the $s$-process models predict $[\mathrm{Xe} / \mathrm{Kr}$ ] values comparable to [hs/ls] values. The observed $[\mathrm{Xe} / \mathrm{Kr}]$ ratios in planetary nebulae can be used to further constrain the total number of free neutrons and consequently the ${ }^{13} \mathrm{C}$ pocket (Sharpee et al. 2007; Pignatari et al. 2008). Furthemore, Rb can be directly seen in planetary nebulae, providing us with a constrain on the initial mass of the parent star and the opportunity to disentangle the effects of hot bottom burning in massive AGB stars (potentially Rb-rich) and of extra mixing in low-mass AGB stars (certainly Rb-poor) on the He and $\mathrm{N}$ excesses observed in Type I planetary nebulae.

From a preliminary analysis by Pignatari et al. (2008), the observations of the sprocess $[\mathrm{Xe} / \mathrm{Kr}]$ in $\mathrm{PNe}$ seem to be consistent with the theoretical predictions of AGB models. However, Sterling et al. (2016) (see Fig. 2) show a mismatch between models and observations for $\mathrm{Kr}$, with the observations being higher than the model predictions. This problem needs to be analyzed in more details, also in the light of the other independent s-process observations of noble gases available from meteoritic presolar silicon carbide (SiC) grains, which formed in the winds of C-rich AGB stars. 

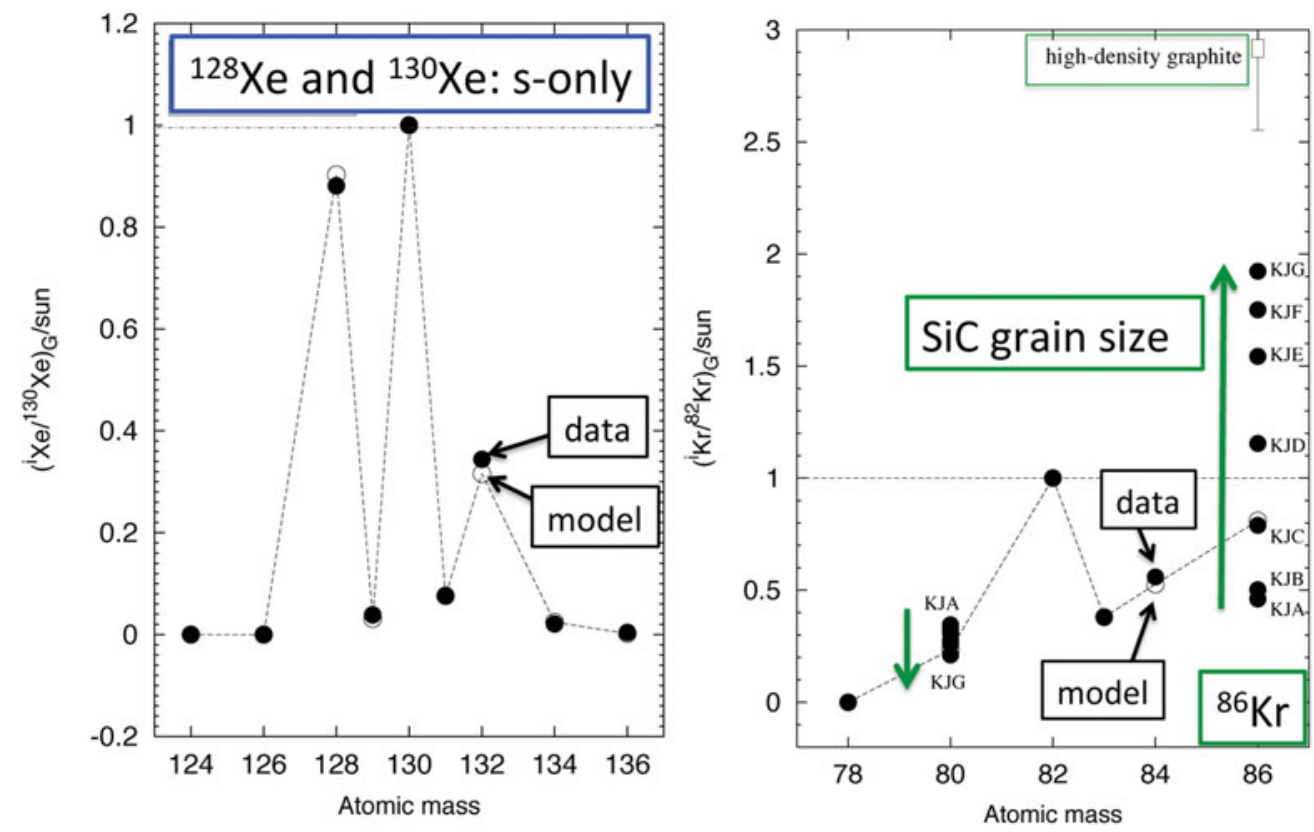

Figure 3. Figures adapted from Lugaro (2005) showing a comparision between the isotopic composition of Xe and $\mathrm{Kr}$ (normalised to ${ }^{130} \mathrm{Xe}$ and ${ }^{82} \mathrm{Kr}$, respectively, and to the solar composition) measured in collections of millions of SiC grains (in bulk by Lewis et al. 1994) and predicted by AGB models for the typical parent stars of the grains (1.5-3 $M_{\odot}$ and solar metallicity).

\subsection{The record of planetary nebula processes in stardust $\mathrm{SiC}$}

Meteoritic stardust SiC grains contain noble gases that carry the signature of processes occurring in AGB stars and planetary nebulae. One of the first evidences that most of these grains originated from the envelopes of C-rich AGB star comes from the composition of the noble gas Xe (left panel of Fig. 3), which shows the indisputable signature of the $s$ process in AGB stars. The Xe isotopic ratios produced by the $s$ process are predominantly controlled by the neutron-capture cross sections of the different Xe isotopes, which strongly favour the production of ${ }^{128} \mathrm{Xe}$ and ${ }^{130} \mathrm{Xe}$. These are the two Xe $s$-only isotopes that are shielded from $r$-process production by their isobars in Te and are predominantly produced by the $s$ process.

Very intriguing is the abundance trend of the different noble gases, $\mathrm{He}, \mathrm{Ne}, \mathrm{Ar}, \mathrm{Kr}$, and Xe with the size of the grains. Since noble gases are chemically inert, they are believed to have been incorporated in the $\mathrm{SiC}$ grains via an implantation process. Verchovsky et al. (2004) analysed the data from Lewis et al. (1994) in the light of an implantation model and discovered that the two different patterns (highlighted in green and blue in Fig. 4) can be reproduced by the models by varying the energy per nucleon, an input parameter of the model. A relatively low energy per nucleon, of the order of $0.5 \mathrm{keV}$, can reproduce the pattern observed in Xe. This energy can be associated with turbulence in the winds during the AGB phase, which have typical speeds of $10-30 \mathrm{~km} / \mathrm{s}$ (the cold component, Table 1). As a consequence, Xe atoms must have been implanted carrying the signature of the matter in the winds of the AGB star. On the other hand, a much higher energy per nucleon $(\simeq 40 \mathrm{keV})$ is required to reproduce the pattern observed in $\mathrm{He}, \mathrm{Ne}$, Ar. This is because if the atom has too much energy, it cannot be stopped inside small grains. Post-AGB/planetary nebula fast winds $(\simeq 700 \mathrm{~km} / \mathrm{s})$ can imprint such an energy in the noble gases atoms (the hot component, Table 1). As a 


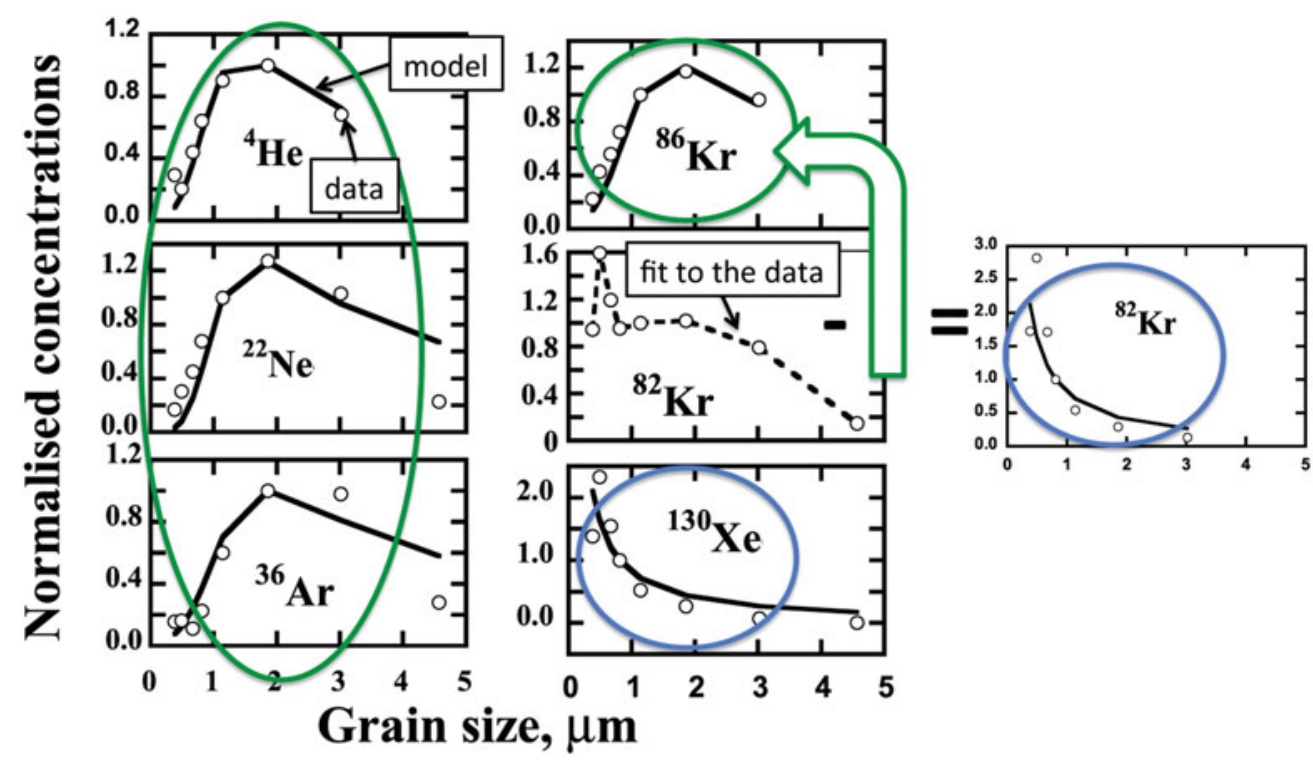

Figure 4. Figure adapted from Verchovsky et al. (2004) (CAAS. Reproduced with permission) and showing a comparison between the abundances of the different noble gases in meteoritic $\mathrm{SiC}$ as a function of the grain size (from Lewis et al. 1994) and the implantation model. Two clearly different patterns can be identified: ${ }^{4} \mathrm{He},{ }^{22} \mathrm{Ne},{ }^{36} \mathrm{Ar}$, and ${ }^{86} \mathrm{Kr}$ are most abundant in large grains (highlighted in green), ${ }^{130} \mathrm{Xe}$ is most abundant in the small grains (highlighted in blue), while the ${ }^{82} \mathrm{Kr}$ abundance is made by a mixture of the two patterns, i.e., the blue pattern can be obtained by subtracting the ${ }^{86} \mathrm{Kr}$ green pattern from the total ${ }^{82} \mathrm{Kr}$.

Table 1. Qualitive description of the two components of noble gases implanted in meteoritic $\mathrm{SiC}$ grains.

\begin{tabular}{|c|c|c|}
\hline Component & cold & hot \\
\hline Grain size $(\mu \mathrm{m})$ & $<1$ & $>1$ \\
${ }^{4} \mathrm{He}$ & no & yes \\
${ }^{22} \mathrm{Ne}$ & no $^{1}$ & yes \\
${ }^{36} \mathrm{Ar}$ & no & yes \\
${ }^{82} \mathrm{Kr}$ & yes & yes \\
${ }^{86} \mathrm{Kr}$ & no & yes \\
${ }^{130} \mathrm{Xe}$ & yes & no \\
Energy/nucleon $(\mathrm{keV})$ & 0.5 & 40 \\
Implantation winds & AGB & post-AGB \\
Wind speed (km/s) & $10-30$ & 700 \\
AGB nucleosynthetic signature & envelope & intershell (final) \\
\hline
\end{tabular}

Notes:

1 Not ionised.

${ }^{2}$ Nucleosynthetic signature not present.

${ }^{3}$ Much less abundant than the cold component.

consequence, $\mathrm{He}, \mathrm{Ne}$, and Ar must have been implanted carrying the signature of matter in the post-AGB winds, the same winds invoked as one of the possible factors involved in the production of planetary nebulea. Finally, $\mathrm{Kr}$ is a mixute of the two components, some of it being implanted in the AGB phase, and some during the planetary nebula phase.

The question arises of why He, Ne and Ar show only the fast wind/planetary nebula component, Xe shows only the AGB wind component, and Kr shows them both. 
Verchovsky et al. (2004) proposed that these signatures are due to the different ionisation energy of the noble gases, which decreases with the number of electrons: Xe has the lowest $(\simeq 12 \mathrm{eV})$ ionization energy, and He the highest $(\simeq 25 \mathrm{eV})$ ionisation energy. If we assume that the atoms must be ionized to be implanted in the grains, then their different ionization energies can fractionate their abundances.

From the observed isotopic ratios of the noble gases we can gather detailed information about the nucleosynthetic signature carried by the two components. The Xe isotopic ratios are matched by the composition predicted in the AGB winds (left panel of Fig. 3). This is the mixture of $s$-process-rich intershell material carried to the envelope by the TDU during the whole AGB phase and the initial composition of the stars, close to the solar system composition. On the other hand, to match the nucleosynthetic signatures presented by $\mathrm{He}$ and $\mathrm{Ne}$, and in particular the so-called Ne-E(H) component extremely enriched in ${ }^{22} \mathrm{Ne}$, almost pure intershell material is required, with no dilution by the original envelope material. Post-AGB winds can carry such almost pure signature only in the case some flavour of a late or very late TP occurred (Blöcker 2001), which affects $\sim 20 \%$ of the AGB population (e.g., Werner \& Herwig 2006). It is possible that a nucleosynthetic signature such as that of the H-rich envelope is also present for $\mathrm{He}$ and $\mathrm{Ne}$ in the hot component, but, it is not seen because its abundance is much lower than that of the intershell in the late or very late TP scenario.

The ${ }^{82} \mathrm{Kr}$ abundance shows both the cold and the hot components (as Kr may be ionised in both cases), while ${ }^{86} \mathrm{Kr}$ shows only the fast wind/planetary nebula hot component. The most likely explanation is that the nucleosynthetic signature of the cold component carries a ${ }^{82} \mathrm{Kr}$ but not a ${ }^{86} \mathrm{Kr}$ excess, while the nucleosynthetic signature of the hot component carries both. The explanation for this difference lies in the fact that ${ }^{82} \mathrm{Kr}$ is located on the main $s$-process path, while ${ }^{86} \mathrm{Kr}$ is produced by the activation of the branching point at ${ }^{85} \mathrm{Kr}$, for neutron densities exceeding few $10^{8} \mathrm{n} / \mathrm{cm}^{3}$. The hot component recorded in large grains points to high ${ }^{86} \mathrm{Kr} /{ }^{82} \mathrm{Kr}$ ratios in the intershell (right panel of Fig. 3). The stellar origin of such signature remains debated. Potential proposed explanations are models of metallicity lower than solar and either a higher efficiency of the activation of ${ }^{13} \mathrm{C}$ pocket (Pignatari et al. 2006) or the ingestion of the ${ }^{13} \mathrm{C}$ pocket inside the TPs (Raut et al. 2013), which can occur if the initial mass is lower than 1.5 $M_{\odot}$. However, such models predict $\mathrm{Sr}$ and $\mathrm{Ba}$ isotopic ratios inconsistent with measurements of the same bulk and single SiC grains (Gallino et al. 1997; Lugaro et al. 2003a; Barzyk et al. 2007; Ávila et al. 2013; Liu et al. 2015). The impact of convective-boundary mixing processes at the bottom of the convective thermal pulse (e.g., Lugaro et al. 2003b; Battino et al. 2016) needs to be also evaluated for Kr and Xe. Another possibility would be that the production of extra ${ }^{86} \mathrm{Kr}$ occurs during the post-AGB phase in association with a late or a very late TP that is already required to carry to the stellar surface the almost pure intershell material seen in the hot component (e.g., as in the case of the intermediate neutron-capture process seen in Sakurai's object; Herwig et al. 2011).

Finally, it would be important to fully define the connection between the excess ${ }^{86} \mathrm{Kr}$ in the stardust data and the Kr excess seen in the planetary nebulae observations (Fig. 2). From a back-of-the-envelope calculation, if we increase by a factor of 4 the ${ }^{86} \mathrm{Kr}$ abundance in the $2.5 M_{\odot}$ model shown in Fig. 2, to simulate an extreme composition such as that shown by the high-density graphite grains (Fig. 3), the $[\mathrm{Kr} / \mathrm{Fe}]$ ratio would increase from its current value of 0.43 to 0.66 , which is a much better match with the data. As we mentioned ealier, the capability of theoretical AGB models to reproduce $\mathrm{PN} s$-process observations of $\mathrm{Kr}$ and $\mathrm{Xe}$ is still matter of debate, and a consistent scenario should be derived that works for both PNe and measurements from presolar grains. 


\section{Summary and conclusions}

Nucleosynthesis in AGB stars shapes the abundances observed in planetary nebulae via a complex interplay of nuclear reactions and mixing processes such as the third dredge-up and hot bottom burning. As such, planetary nebula abundances provide an independent, complementary testbed for all these processes, including the production of the elements heavier than rion via the $s$ process. Conversely, the observed chemical abundance can be used to constraint the evolutionary path leading to the formation of planetary nebulae and the initial mass range. Specifically, we confirm that observations of $[\mathrm{Xe} / \mathrm{Kr}]$ are a potential proxy for the traditional [hs/ls] $s$-process index and that the abundance of $\mathrm{Rb}$ can provide us crucial further constraints on the initial masses of planetary nebulae and the nucleosynthesis and mixing processes in massive AGB stars. Furthermore, noble gas abundances observed in meteoritic stardust grains are tracers of processes in planetary nebulae, from ionisation and implantation, to nucleosynthesis in the central star. The combination of all these different independent constraints will allow us to open new ways to understand planetary nebulae and the link to their parent AGB stars.

\section{References}

Abia, C., Busso, M., Gallino, R., Domínguez, I., Straniero, O., \& Isern, J. 2001, Astrophys. J., 559,1117

Abia, C., Cunha, K., Cristallo, S., \& de Laverny, P. 2015, Astron. Astrophys., 581, A88

Arlandini, C., Käppeler, F., Wisshak, K., Gallino, R., Lugaro, M., Busso, M., \& Straniero, O. 1999, Astrophys. J., 525, 886

Ávila, J. N., Ireland, T. R., Gyngard, F., Zinner, E., Mallmann, G., Lugaro, M., Holden, P., \& Amari, S. 2013, Geochim. Cosmochim. Acta, 120, 628

Barzyk, J. G., Savina, M. R., Davis, A. M., Gallino, R., Gyngard, F., Amari, S., Zinner, E., Pellin, M. J., Lewis, R. S., \& Clayton, R. N. 2007, Meteoritics and Planetary Science, 42, 1103

Battino, U., Pignatari, M., Ritter, C., Herwig, F., Denisenkov, P., Den Hartogh, J. W., Trappitsch, R., Hirschi, R., Freytag, B., Thielemann, F., \& Paxton, B. 2016, Astrophys. J., 827, 30

Bisterzo, S., Travaglio, C., Gallino, R., Wiescher, M., \& Käppeler, F. 2014, Astrophys. J., 787, 10

Blöcker, T. 2001, Astrophys. Space Sci., 275, 1

Cristallo, S., Straniero, O., Gallino, R., Piersanti, L., Domínguez, I., \& Lederer, M. T. 2009, Astrophys. J., 696, 797

Cristallo, S., Straniero, O., Piersanti, L., \& Gobrecht, D. 2015, Astrophys. J. Suppl., 219, 40

Gallino, R., Arlandini, C., Busso, M., Lugaro, M., Travaglio, C., Straniero, O., Chieffi, A., \& Limongi, M. 1998, Astrophys. J., 497, 388

Gallino, R., Busso, M., \& Lugaro, M. 1997, in American Institute of Physics Conference Series, Vol. 402, American Institute of Physics Conference Series, ed. T. J. Bernatowicz \& E. Zinner, $115-153$

Goriely, S. \& Mowlavi, N. 2000, Astron. Astrophys., 362, 599

Herwig, F. 2005, Ann. Rev. Astron. Astrophys., 43, 435

Herwig, F., Pignatari, M., Woodward, P. R., Porter, D. H., Rockefeller, G., Fryer, C. L., Bennett, M., \& Hirschi, R. 2011, Astrophys. J., 727, 89

Karakas, A. I. \& Lattanzio, J. C. 2014, Publ. Astron. Soc. Aust., 31, 30

Karakas, A. I. \& Lugaro, M. 2016, Astrophys. J., 825, 26

Lewis, R. S., Amari, S., \& Anders, E. 1994, Geochim. Cosmochim. Acta, 58, 471

Liu, N., Savina, M. R., Gallino, R., Davis, A. M., Bisterzo, S., Gyngard, F., Käppeler, F., Cristallo, S., Dauphas, N., Pellin, M. J., \& Dillmann, I. 2015, Astrophys. J., 803, 12

Lugaro, M. 2005, Stardust from meteorites. An introduction to presolar grains Copyright@2005 World Scientific 
Lugaro, M., Davis, A. M., Gallino, R., Pellin, M. J., Straniero, O., \& Käppeler, F. 2003a, Astrophys. J., 593, 486

Lugaro, M., Herwig, F., Lattanzio, J. C., Gallino, R., \& Straniero, O. 2003b, Astrophys. J., 586, 1305

Lugaro, M., Karakas, A. I., Stancliffe, R. J., \& Rijs, C. 2012, Astrophys. J., 747, 2

Lugaro, M., Ugalde, C., Karakas, A. I., Görres, J., Wiescher, M., Lattanzio, J. C., \& Cannon, R. C. 2004, Astrophys. J., 615, 934

Pignatari, M., Gallino, R., Amari, S., \& Davis, A. M. 2006, Mem. Soc. Astron. It., 77, 897

Pignatari, M., Gallino, R., Cristallo, S., \& Straniero, O. 2008, in American Institute of Physics Conference Series, Vol. 1001, Evolution and Nucleosynthesis in AGB Stars, ed. R. Guandalini, S. Palmerini, \& M. Busso, 154-159

Pignatari, M., Herwig, F., Hirschi, R., Bennett, M., Rockefeller, G., Fryer, C., Timmes, F. X., Ritter, C., Heger, A., Jones, S., Battino, U., Dotter, A., Trappitsch, R., Diehl, S., Frischknecht, U., Hungerford, A., Magkotsios, G., Travaglio, C., \& Young, P. 2016, Astrophys. J. Suppl., 225, 24

Raut, R., Tonchev, A. P., Rusev, G., Tornow, W., Iliadis, C., Lugaro, M., Buntain, J., Goriely, S., Kelley, J. H., Schwengner, R., Banu, A., \& Tsoneva, N. 2013, Physical Review Letters, 111,112501

Roberts, L. F., Woosley, S. E., \& Hoffman, R. D. 2010, Astrophys. J., 722, 954

Sharpee, B., Zhang, Y., Williams, R., Pellegrini, E., Cavagnolo, K., Baldwin, J. A., Phillips, M., \& Liu, X.-W. 2007, Astrophys. J., 659, 1265

Sterling, N. C., Dinerstein, H. L., Kaplan, K. F., \& Bautista, M. A. 2016, Astrophys. J. Lett., 819, L9 Title: Discovery of Rubidium, Cadmium, and Germanium Emission Lines in the Nearinfrared Spectra of Planetary Nebulae, http://iopscience.iop.org/article/10.3847/2041$8205 / 819 / 1 /$ L9/meta

Thielemann, F.-K., Arcones, A., Käppeli, R., Liebendörfer, M., Rauscher, T., Winteler, C., Fröhlich, C., Dillmann, I., Fischer, T., Martinez-Pinedo, G., Langanke, K., Farouqi, K., Kratz, K.-L., Panov, I., \& Korneev, I. K. 2011, Progress in Particle and Nuclear Physics, 66,346

van Raai, M. A., Lugaro, M., Karakas, A. I., García-Hernández, D. A., \& Yong, D. 2012, Astron. Astrophys., 540, A44

Verchovsky, A. B., Wright, I. P., \& Pillinger, C. T. 2004, Astrophys. J., 607, 611 Title: Astrophysical Significance of Asymptotic Giant Branch Stellar Wind Energies Recorded in Meteoritic SiC Grains http://iopscience.iop.org/article/10.1086/383230/meta

Werner, K. \& Herwig, F. 2006, Publ. Astron. Soc. Pac., 118, 183

Zamora, O., García-Hernández, D. A., Plez, B., \& Manchado, A. 2014, Astron. Astrophys., 564, L4

\section{Discussion}

Muthumariappan: Ne abundances in the H-poor knots of A30, A78 are several percentage more than expected from AGB nucleosynthesis. How do we account the same?

De MARCO: There are too many Type I PN to be explained by hot bottom burning, i.e. stars with initial mass $>4 \mathrm{M} \odot$.

LUGARO: We think there could be processes to increase N/O even in lower mass stars. The $\mathrm{Rb}$ abundance is a better indicator of mass. Finding low Rb (i.e. lower mass) and high $\mathrm{N} / \mathrm{O}$ would be an indication that such additional processes operate (even if we do not yet know what might be). 